\title{
Model for Space Charge Evolution and Dose in Irradiated Insulators at High Electric Fields
}

\author{
A. R. Frederickson \\ PL-GPSP, USAF Phillips Lab, Hanscom AFB, MA 01731-3010 \\ S. Woolf \\ ARCON Corp., 260 Bear Hill Rd., Waltham, MA 02154 \\ J. C. Garth \\ PL-VTER, USAF Phillips Lab, Hanscom AFB, MA 01731-3010
}

\begin{abstract}
A new method for calculating dose, charge deposition, current and electric field profiles across a dielectric slab irradiated by 1-3 MeV electron beams has been developed. The model consists of following the electric field build-up with time. A sequence of Monte Carlo calculations for relativistic electrons moving in an electric field determines the motion of the high-energy electrons. This is coupled with electric field solutions using a 1-D electrostatic field code. As time proceeds, the profile of high-energy electron current changes as well as the dose deposition which affects the magnitude of the conduction currents. Several mechanisms for conduction in the dielectric are taken into account. Our model predictions are compared with Kerr effect electric field data on irradiated polymethylmethacrylate (PMMA) obtained by Hikita et al. (1988).
\end{abstract}

\section{INTRODUCTION}

Theoretical models of space charge evolution and dose in irradiated insulators at high electric fields can be improved by the inclusion of the effects of the electric field on the fast electron scattering. Consideration of these effects impacts on the development of the dose, charge, fast electron current and charge deposition. In this paper, each of these terms is calculated in computer simulation with its full time and spatial dependence.

New measurements by Hikita et al.[1] provide electric field and charging data on insulators irradiated by high energy $(\mathrm{HE})$ electron beams. Previous predictions of electric field and charge distributions as a function of time have not included the effects of the electric field on the motion of the $\mathrm{HE}$ electrons[2,3]. For electrons well below $100 \mathrm{keV}$, where the material stopping power dominates, the field effects are less important. It has been observed for electrons above 1 $\mathrm{MeV}$ that their trajectories are foreshortened in charged insulators[4-6].

Nearly all of the Hikita data had one striking difference from the previously published theoretical data for partially penetrating electron beams. In the old theoretical data the charge centroid was predicted to move away from the surface through which the monoenergetic beam entered as irradiation progressed. Hence the old theory predicted a much deeper depth for the charge centroid than the Hikita data showed.

Additionally the old theory predicted only a small sharpening of the spatial distribution of stopped electrons as time progressed. Instead Hikita et al. measured that the centroid of charge moved rapidly toward the entrance surface and developed a sharply defined peak of stopped electrons. It was obvious that the effect of the electric field on the $\mathrm{HE}$ electrons must be included in any good theory.

We have found that taking into account the electric field build-up in our Monte Carlo simulation on the motion of the HE electrons gives better agreement with Hikita's experiments than do the older models. As a by-product of this improved modeling, better estimates of the radiationinduced-conductivity (RIC) can be made by comparison with the measurements. We also find that an electric fieldenhanced conductivity is needed to explain the electric fields measured at the longer irradiation times.

This modeling provides the capability for characterizing charge storage in insulators with perhaps a factor of two improvement in accuracy. Old measurements of RIC are in error because they ignore the field effects on $\mathrm{HE}$ electrons. In addition, estimates of radiation-induced currents in the leads of irradiated capacitors may be in substantial error if this effect is not considered. Moreover, inclusion of this effect in modeling will usually result in much larger electric field predictions, thereby providing improved warning of approach to breakdown threshold. This model also provides an improved basis for understanding the breakdown of spaceborne electronic insulation due to $\mathrm{HE}$ electron radiation belts.

The experimental method developed by $M$. Zahn[1] provides a turning point for this field of study.

\section{MODEL DESCRIPTION}

\section{A. Introduction}

Recent electric field and space charge measurements[1] as a function of time in electron beam irradiated insulators provide an opportunity to improve previously developed 
theoretical models[2,3]. Theoretical estimates of the space charge and internal electric fields were obtained in these older models using the following procedure (the "NUMIT" algorithm[7]):

1) estimate the initial radiation-induced $\mathrm{HE}$ electron current, $J_{R}(z)$ and dose rate profile $D(z)$, either directly from Monte Carlo data or using a modification to Tabata's[8] energy deposition profile algorithm;

2) integrate the continuity equation for current and charge over a short irradiation time interval, $\Delta t$, from time $t$ $\Delta t$ to $t$ to obtain the charge density $\rho(z, t)$ at time $t$. The time interval $\Delta t$ is held sufficiently small to allow for valid numerical integration (i.e. it is assumed that $J$ and $\rho$ do not vary much over this interval).

3 ) use the integral form of Coulomb's law to solve for the internal electric field $E(z, t)$;

4) determine the depth dependence of the conductivity in the insulator based on the dose rate profile and the conduction physics in the insulator;

5) determine the conduction current profile based on (3) and (4) above and add the conduction current to the radiation-induced current in (1) above to obtain the total current profile in the presence of the field $E(z)$;

6) with the total current, return to step (2) above and iterate through the process, steps (2-5), for another time interval. Repeat the iteration as often as necessary.

Our improved model changes this procedure as follows: A Monte Carlo simulation of the transport of $\mathrm{HE}$ electrons in the insulator under the influence of the electric field is performed to periodically recalculate $J_{R}(z)$ and dose rate profile $D(z)$. Instead of using only an initial estimate of $J_{R}(z)$ and $D(z)$ at step (1), after every 100 or more $\Delta t$, i.e. steps (2$6)$, we interrupt the iteration and recalculate $J_{R}(z)$ and $D(z)$ using the electric field at this time. We then resume steps(2$6)$ and interrupt again after $100 \Delta t$ steps to calculate $J_{R}(\mathrm{z})$ and $D(z)$ again. In practice we typically recalculate $J_{R}(\mathrm{z})$ and $D(z)$ about 20 times for 20 different electric fields.

We have thus replaced the field-independent Monte Carlo calculation or modified Tabata algorithm by a sequence of Monte Carlo calculations with electric field included. Our new Monte Carlo algorithm is described in the next section.

\section{B. Monte Carlo Model}

The Monte Carlo calculation yields the current and dose profiles, $J(\mathrm{z})$ and $D(\mathrm{z})$, with a full simulation of the $\mathrm{HE}$ electron transport. The algorithm used is based on Berger's[9] formulation of the Goudsmit-Saunderson multiple scattering distribution and is similar to that found in the ITS[10] code system. Our algorithm departs from Berger's basic method by: 1) incorporating the relativistic equations of motion[11] for an HE electron in the presence of an electric field; and 2) adjusting the path length step between scatters to take into account the effective modification of the stopping force on the electron by acceleration or deceleration due to the electric field.

\section{Electron Trajectory Calculations}

Our Monte Carlo simulation of electron $(\sim 10 \mathrm{keV}-10$ $\mathrm{MeV}$ ) transport in insulators applies to transport in a single material layer in slab geometry, a much less ambitious undertaking than the more general codes[10,12]. The electron trajectory is simulated by a series of inter-collision segments. In a given trajectory segment, it is assumed that the electron travels a distance $\Delta s$ from point 1 to point 2 in the insulating medium (see Fig. 1).

The stopping power force acts along $\Delta s$ (arbitrary direction), and it is assumed that the electric field force acts along the $z$-direction. The braking force, due to the action of the stopping power, is defined as $\vec{F}$, with magnitude equal to

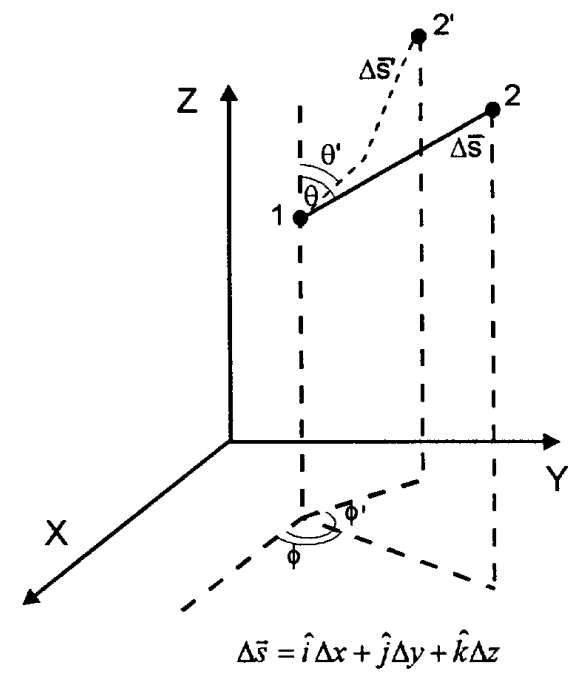

Fig. 1. Coordinate frame for electron Monte Carlo calculations

the stopping power $(|\vec{F}|=|d W / d s|)$ and with components $F_{x}, F_{y}, F_{z}$. If the initial components of the electron momentum $\vec{p}_{0}$ are given by $p_{x 0}, p_{y 0}, p_{z 0}$, the trajectory equations for the electron are

$x(t)=\frac{c F_{x}}{a^{2}}\left\{\left[a^{2} t^{2}+h t+g^{2}\right]^{1 / 2}-g\right\}+c\left[p_{x 0}-\frac{F_{x} h}{2 a^{2}}\right] I_{1}(t)$

$y(t)=\frac{c F_{y}}{a^{2}}\left\{\left[a^{2} t^{2}+h t+g^{2}\right]^{1 / 2}-g\right\}+c\left[p_{y 0}-\frac{F_{y} h}{2 a^{2}}\right] I_{1}(t)$,

$z(t)=c p_{z o} I_{1}(t)+c\left(e E+F_{z}\right) I_{2}(t)$,

where 


$$
\begin{aligned}
& I_{1}(t) \equiv \frac{1}{a} \log \left\{\frac{2 a\left[a^{2} t^{2}+h t+g^{2}\right]^{1 / 2}+2 a^{2} t+h}{2 a g+h}\right\}, \\
& I_{2}(t) \equiv \frac{1}{a^{2}}\left\{\left[a^{2} t^{2}+h t+g^{2}\right]^{1 / 2}-g\right\}-\frac{h}{2 a^{2}} I_{1}(t), \\
& \text { and } \\
& a^{2}=(e E)^{2}+2 e F_{z}+F_{x}^{2}+F_{y}^{2}+F_{z}^{2}, \\
& h=2\left[p_{x 0} F_{x}+p_{y 0} F_{y}+p_{z 0} F_{z}+p_{z 0} e E\right], \\
& g^{2}=p_{z 0}^{2}+p_{x 0}^{2}+p_{y 0}^{2}+m_{0}^{2} c^{2} .
\end{aligned}
$$

\section{Effect of Electric Field on Determination of Intercollision Path Length}

The standard method (due to Berger) for determining the path length step $\Delta s_{n}$ between collisions $n$ and $n+1$ with no electric field present is

$$
\Delta s_{n}=\frac{\Delta W_{n}}{-|d W / d s|} .
$$

where $\Delta W_{n}\left(=W_{n}-W_{n+1}\right)$ is the pre-determined energy loss between multiple collisions, i.e. $W_{n+1}=.9170 W_{n}$ For a given $\Delta W_{n}$, with an electric field present, we modify $\Delta s_{n}$ as follows

$$
\Delta s_{n}=\frac{\Delta W_{n}}{-|d W / d s|+e E \cos \theta} .
$$

Thus, if the direction of $E$ is such as to oppose motion of the electron in the positive $z$-direction, the effective stopping power is increased (in magnitude) and $\Delta s_{n}$ is decreased. Conversely, if the field direction assists the motion of the electron along $z, \Delta s_{n}$ could be lengthened. In the actual Monte Carlo program it is necessary to maintain a path length step approximation which is at least as accurate as that used in the original zero field algorithm. To achieve this the value of $\Delta s_{n}$ is not allowed to exceed the value that would be adopted in the absence of an electric field. To test the accuracy of our Monte Carlo algorithm, we performed benchmark calculations[13] to compare with exact solutions of the electron trajectory equations for the special cases of: 1) non-zero stopping power; zero angular scattering deflection; $\vec{E}, \vec{p}_{0}$ directed along $z$; and 2) zero stopping power; zero angular scattering deflection; $\frac{\vec{E} \cdot \vec{p}_{0}}{|\vec{E}|\left|\vec{p}_{0}\right|}=1 / \sqrt{2}$.

\section{I-D Electrostatic Field Code (NUMIT)}

The electrostatic field code, NUMIT[2,7], provides theoretical estimates of the space charge, current density and electric fields internal to the 1-D dielectric. It does so by iteratively solving the coupled equations

$$
\begin{aligned}
& \frac{\partial \rho(z, t)}{\partial t}=-\frac{d J(z, t)}{d z}, \quad \text { (eqn. of continuity) } \\
& \varepsilon \frac{d}{d z} E(z, t)=\rho(z, t) \\
& \text { and }
\end{aligned}
$$

$J(z, t)=J_{R}(z, t)+J_{C}[E(z, t) ; D(z, t) ; \ldots]$,

where $J_{C}(z, t)$ is the conduction, diffusion and any other low energy charge current density, $\varepsilon$ is the dielectric permittivity, $\rho(z, t)$ is the net space charge density,

with known initial conditions $E(z, 0) ; \rho(z, 0) ; J(z, 0)$ and boundary conditions of fixed total voltage applied across the sample of constant dimension.

NUMIT is a simple code, but its capabilities are quite broad. It has been used to follow the time evolution of the development of the depletion region in $n^{+} n$ semiconductor junctions. It has modeled the currents in an irradiated fibrous pad where conduction is controlled by percolation theory. And of course, it has modeled irradiated solid insulators.

One may internupt the program NUMIT at any arbitrary time $t_{i}$. Keeping the resulting $E\left(z, t_{i}\right)$ and $\rho\left(z, t_{i}\right)$ as initial conditions, the calculation can resume with changed conditions on: $J\left(z, t_{i}\right)$, applied voltage and material parameters including dielectric constant, conductivity, and density.

For comparison to Hikita we periodically interrupt NUMIT in order to change the HE electron current $J_{R}\left(z, t_{i}\right)$,

and the conductivity due to radiation $\sigma_{R}(z, t)=k_{p} D^{\Delta}(z, t) . D$ is the dose rate[rads/s] and $k_{p}$ is the RIC coefficient. We choose the time of interruption $t_{i}$ so that the electric field $E\left(z, t_{i}\right)$ produces only a small change to $J_{R}\left(z, t_{i}\right)$ and to $D\left(z, t_{i}\right)$ relative to the values prior to $t_{i}$. By frequently interrupting NUMIT, we can make the calculation into a quasi-continuous function of the now time dependent functions $J_{R}(z, t)$ and $D(z, t)$. These functions are thereby dependent on the electric field as long as one interrupts NUMIT sufficiently often.

We used the standard popular form[15-17] for conductivity current $J_{C}$ which is given by

$J_{C}(z, t)=\left[k_{p} D^{\Delta}(z, t) E(z, t)+\sigma_{\text {dark }} E(z, t)\right]\left[1+\sinh \frac{q \lambda|E|}{2 k T}\right]$. 
$k_{p}$ is a material-specific coefficient which results from the long range trap modulated mobility of the electrons and holes excited by $\mathrm{HE}$ radiation. $k_{p}$ is determined empirically. $\Delta$ generally lies between 0.5 and 1 , but extreme cases of $\Delta \geq 4$ occur for special materials. This also depends upon trap energy level distributions in the material. For this work we chose $\Delta=0.8$, based on Hikita's published data[1]. $\sigma_{\text {dark }}$ is the standard dark conductivity which is empirically determined. The $(1+\sinh )$ term is high field conductivity enhancement and is derived from field assisted tunneling of carriers from traps. $\lambda$ is empirically determined and reflects the density of traps

The total charge current $J$ is the sum of conduction current $J_{C}$ plus HE electron current $J_{R}$ and is applied in the equation of continuity given earlier. The $\mathrm{HE}$ electron current $J_{R}$ is obtained from the field dependent Monte Carlo at those times when NUMIT is interrupted.

\section{Sample Monte Carlo/NUMIT Results}

The experiment[1] consisted of an electron beam irradiation of a polymethylmethacrylate (PMMA) block of width $d$ with grounded surfaces. Our Monte Carlo calculation included the effect of electron scattering in the accelerator's aluminum window and in the surrounding air.

Fig. 2 shows the time evolution of the dose profile calculated by the Monte Carlo method for a beam energy of $2.5 \mathrm{MeV}$. The curves show that as time proceeds and as the charge in the dielectric builds up, the electron trajectories and the dose curves are pushed to the left, so that the dose profile has roughly half the penetration into the slab at 10 seconds than it did initially. It should be noted that the profile of RIC is approximately proportional to the dose curve, so that the peak of maximum conductivity moves toward the incident surface.

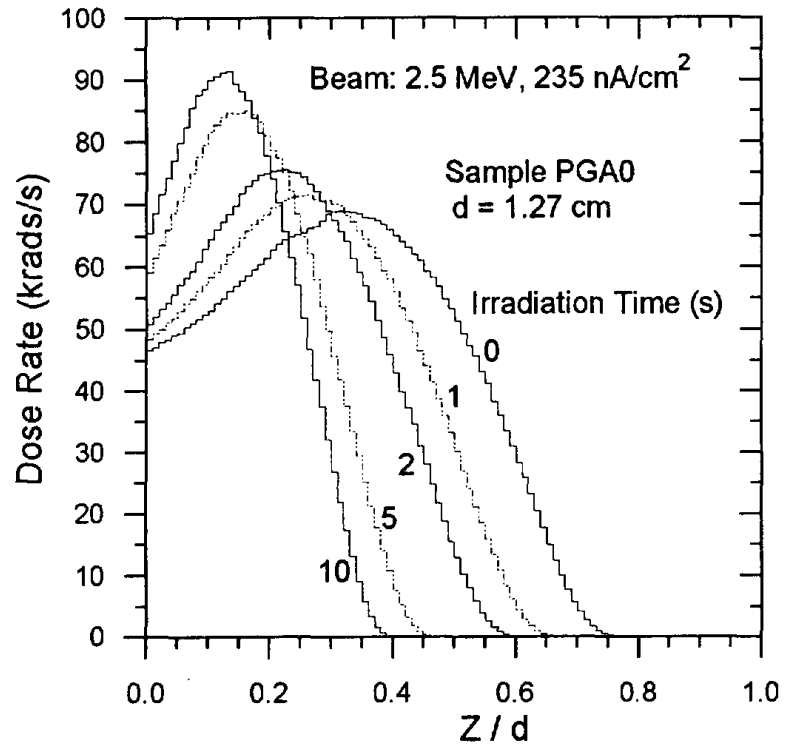

Fig. 2. Time evolution of dose
Fig. 3 shows the time evolution of the charge deposition of $\mathrm{HE}$ electrons and illustrates how the build-up of electric field produces a progressive shift to the left of the charge introduced by the HE electrons.

To repeat our central point, the shift of the HE electron distributions with electric field has not been taken into account in previous theory. The dose, HE charge deposition and $\mathrm{HE}$ current profiles were assumed unchanged with time. The brunt of the effects on replacement current and other observables was assumed to be performed by the conduction electrons. The new "revelation" by Hikita et al. is that there is a very strong build-up of electron charge in a thin plane in the dielectric. This acts to repel the penetration of electrons introduced by the electron beam at later times.

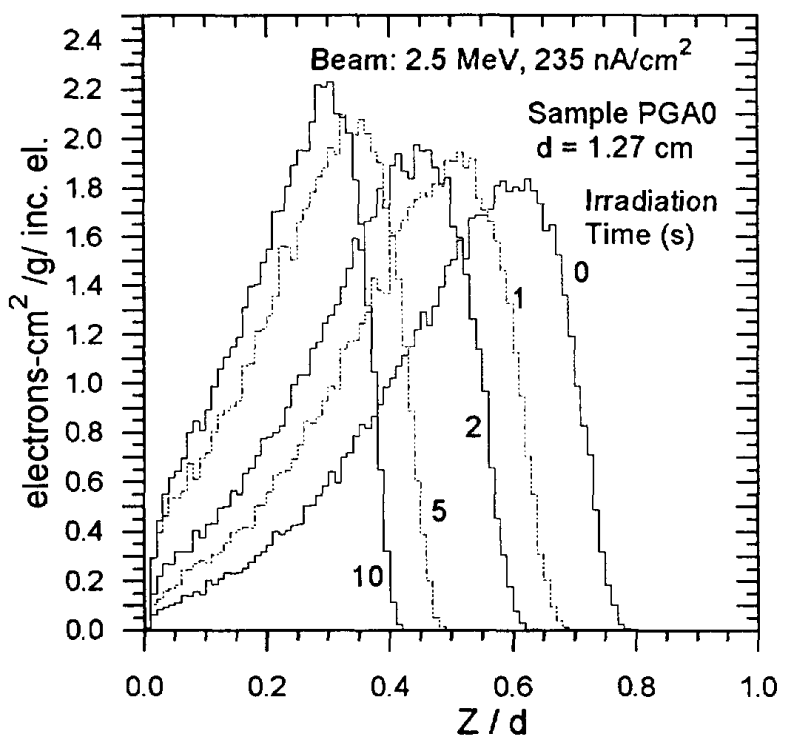

Fig. 3. Time evolution of charge deposition by stopped HE electrons alone. (Stopped conduction charge is not included in this graph.)

Fig. 4 depicts how inclusion or neglect of electric field effects on the motion of the HE electrons affect our predictions for electron beam irradiated dielectrics. Fig. 4 shows HE current profiles at 0 seconds (curves $a$ and $b$ ) and 10 seconds $(c)$. Curve $a$ is calculated using an empirical fit based on the Tabata algorithm[7] and is used in our calculations to represent the results of Monte Carlo without electric field effects on the electron motion. Curve $b$ is essentially the same curve, but calculated with our Monte Carlo method; the slight difference in shape is attributable to the fact that our Monte Carlo model neglects electron straggling, while the Tabata curves are fits to more complete Monte Carlo calculations that incorporate straggling. Curve $d$ is the total current ( $\mathrm{HE}$ plus conduction current) at 10 seconds (using old NUMIT and Tabata's algorithm) which shows the current distribution when electric field effects on 
the HE electron motion are neglected. Electrons penetrate too deeply because the electric field due to the charge buildup does not retard the $\mathrm{HE}$ electrons. Curves $c$ and $e$ show what happens when electric field effects are taken into account in the fast electron motion. The $\mathrm{HE}$ current profile at 10 seconds $(c)$ is greatly pushed toward the surface from the 0 second value $(b)$ and, due to the highly dose-driven increased conductivity toward the incident surface, curve $(e)$ shows that the conduction current greatly cancels $\mathrm{HE}$ current, providing a greatly reduced total current. The sample comes to steady state faster in the new model than in the old model.

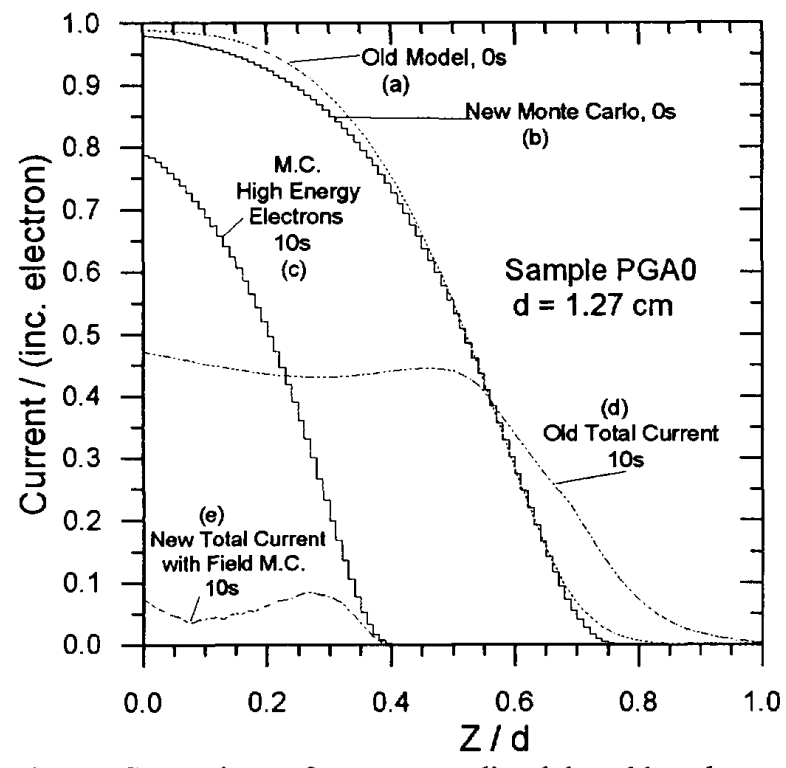

Fig. 4. Comparison of current predicted by old and new models: (a) old model high-energy electrons(HE) at 0s; (b) new model $\mathrm{HE}$ at $0 \mathrm{~s}$; (c) new model $\mathrm{HE}$ at 10s; (d) old model $\mathrm{HE}+$ conduction(CE) at 10s; (e) new model $\mathrm{HE}+\mathrm{CE}$ at $10 \mathrm{~s}$.

\section{EXPERIMENT}

The experimental method[1] is new. It is based upon the Kerr effect wherein the classical optical constant of a dielectric changes proportional to the E-field stress. The shift of the optical constant is measured using a laser interferometer and the E-field is thereby deduced. The PMMA samples are described and numbered in Reference 1.

\section{A. Choice of Beam Energy}

Fig. 5 shows a plot of electric field vs. distance calculated theoretically (using our best values of RIC) at 3 beam energies, 2.4, 2.5, and $2.6 \mathrm{MeV}$ and compared with experimental data on sample PGA0, all at 1.89 seconds. The value of the beam energy that best matched the experimental electric field cross over point (charge centroid) in the center of the sample was also $2.5 \mathrm{MeV}$. This was taken to be the actual electron beam energy used to irradiate the slab, and was used in all our calculations for this set of experiments. It is interesting that Hikita's results, combined with our model, indicated that the van de Graaff voltmeter reading of 2.6 $\mathrm{MeV}$ was actually $2.5 \mathrm{MeV}$. Subsequent calibrations verified this. Further discussion is deferred to section VII below.

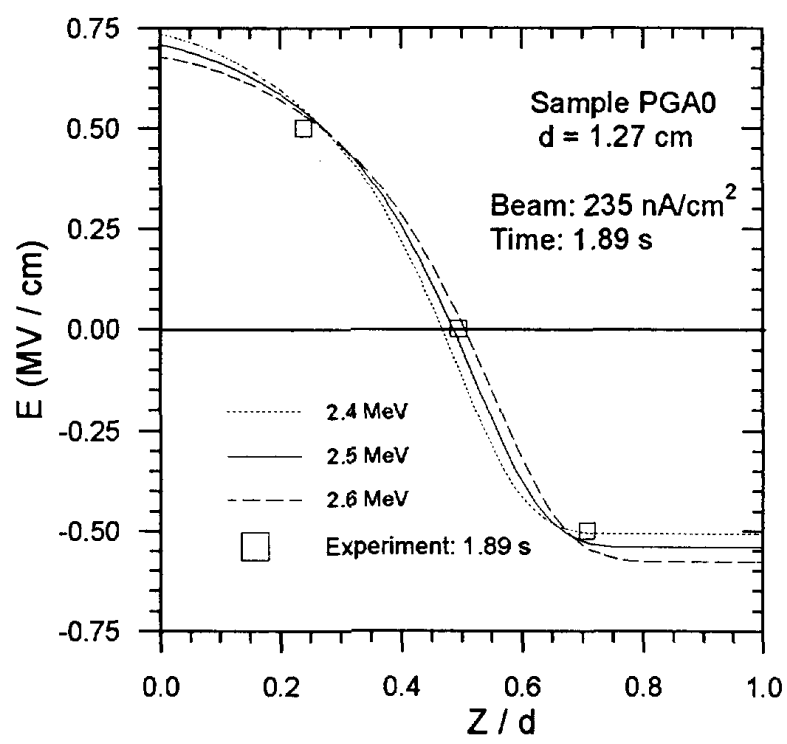

Fig. 5. E-field vs. distance in PMMA for sample PGA0 for 3 incident beam energies. Electron beam current density = $235 \mathrm{nA} / \mathrm{cm}^{2}$. The best match with the experimental zero-field point occurs with $2.5 \mathrm{MeV}$.

\section{B. Determination of "True" Beam Current Density}

For a specified incident beam current density, our model predicts a set of electric field curves for a succession of different irradiation times which can be compared with the experimental electric field data at these same times. Review of the notebook of Hikita and recalibration of the Van de Graaff indicate that our corrections to the beam current are reasonable. The incident beam current was adjusted to give the best overall "fit" at early irradiation time ( 1.89 seconds). In other words, if the general magnitudes of the electric fields were greater than those measured experimentally, the model current density was decreased until the magnitudes of the curves were comparable. The choice of early time minimizes interference on the calibration by conduction processes associated with electric field build-up. 


\section{CAlCUlational PROCEDURE}

Depending on the magnitude of the beam current density, the Monte Carlo and NUMIT runs were made as follows:

1) An initial Monte Carlo calculation was made at 0 seconds irradiation time to determine the original dose and HE current profiles.

2) This information was supplied to the NUMIT code. Time steps of $\sim 0.001$ seconds were used between successive calculations of charge density and electric field. NUMIT was run for around 100 time steps to a time such as 0.1 seconds.

3) A Monte Carlo run of 50000 histories, taking around 20-30 minutes on a personal computer $(486-50 \mathrm{MHz})$, incorporating the electric field supplied by NUMIT in step 2, was made.

4) The new HE dose and current profiles are entered into NUMIT replacing the original ones. NUMIT runs for another 100 time steps to further develop the electric field $E$ and charge density $\rho$.

5) Successive iterations, up to a maximum irradiation time of $\sim 5$ seconds (depending on the beam current density) were made using $\sim 20$ successive Monte Carlo runs. The electric fields plotted in the figures were obtained by NUMIT for times equal to the irradiation times desired, such as 1.89 , 3.99 and 5.76 seconds for sample PGA0.

\section{COMPARISON OF THEORY AND EXPERIMENT FOR ONE CASE}

\section{A. Predictions with Old Model}

Electric field profiles were obtained by performing calculations of $\mathrm{HE}$ current and dose profiles using a modified Tabata algorithm with calculations of the electric field buildup with time. We shall call the use of the Tabata/NUMIT algorithm (equivalent to conventional Monte Carlo without electric fields) the "old" model. This procedure neglects the effect of charge build-up on the motion of the HE electrons.

Consider Fig. 6 which shows the old calculation of electric field as a function of position at three irradiation times, and the corresponding set of experimental points (point symbols as shown in the legend). A RIC coefficient $k_{p}=2.76 \times 10^{-16} \mathrm{sec} / \mathrm{ohm}-\mathrm{m}-\mathrm{rad}$ was used. These old calculations show zero field crossing points that move away from the incident surface with time, in disagreement with experiment, which shows instead a motion of the zero field crossing point toward the incident surface. (Note: the zero field crossing points are believed to be the most accurate of the experimental data points - electric field values as obtained from fringe patterns.) This is the major point of disagreement of the old model with experiment. In Fig. 6 we introduce further a high field conductivity enhancement factor $[1+\sinh (q \lambda|\vec{E}| / 2 k T)]$. This tends to lower the electric field at the incident surface at later times.
To reiterate the key point here, the principal disagreement with experiment is the reversal of the motion of the electric field zero crossing point.

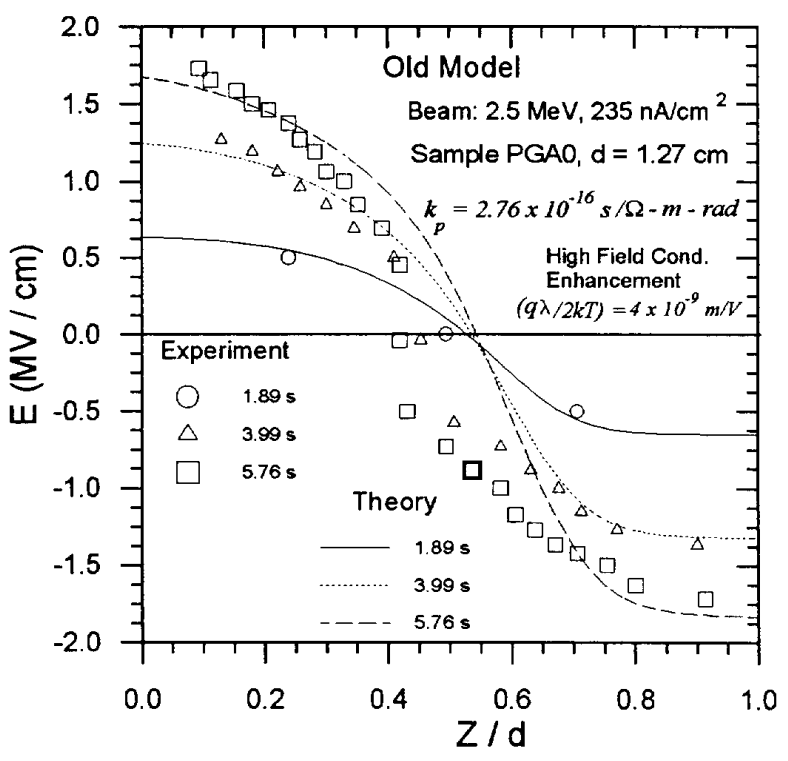

Fig. 6. Electric field vs. distance for sample PGA0. Old model. with $k_{p}=2.76 \times 10^{-16} \mathrm{~s} / \Omega-\mathrm{m}-\mathrm{rad}$.

\section{B. Predictions with Electric Field Monte Carlo}

In contrast with the results shown in Fig. 6, the electric field profiles were obtained by performing a succession of calculations of HE current and dose profiles using our Monte Carlo algorithm, including the effects of the electric field on the motion of the HE electrons, alternated with calculations of the electric field using the NUMIT code. This will be called the "new" model.

In Fig. 7a we calculated the electric field as a function of position at three irradiation times again using (comparing with Fig. 6) a RIC coefficient $k_{p}=2.76 \times 10^{-16} \mathrm{sec} / \mathrm{ohm}-\mathrm{m}-$ rad. Unlike our old model, our new model agrees with experiment, as it predicts motion of the zero field crossing points toward the incident surface with time, showing the effect of the repulsion of the beam by the net negative charge built up in the dielectric.

Figs. 7a,b are comparisons of our new model with the same experimental data. The difference between these two figures is that in Fig. $7 \mathrm{~b}$ a high field conductivity enhancement factor is added. This lowers the electric field at the incident surface, particularly at 5.76 seconds. It is expected that the need for a field-enhanced RIC will be more pronounced as irradiation times and charge build-up increase beyond 5.76 seconds; in general, without inclusion of this enhancement, the model tends to predict a higher slope at the incident surface than seen experimentally. The point is that with our improved model and (hopefully) improved 
experimental electric field measurements, we see the possibility of detecting this field-enhanced RIC effect.

In summary, Figs. 7a,b show excellent agreement with experiment with respect to the motion of the zero electric field crossing point.

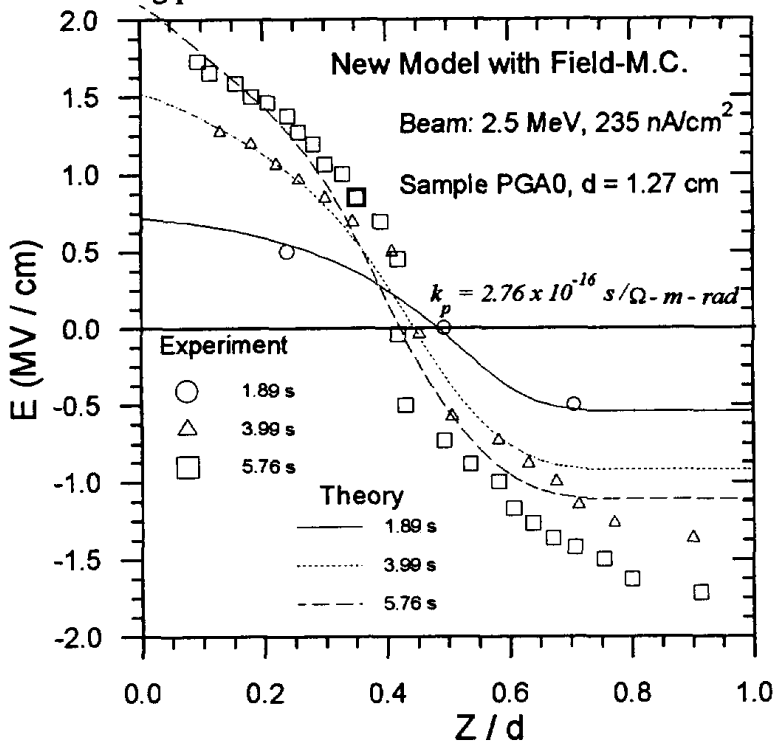

Fig. 7a. Electric field vs. distance for sample PGA0. New model without high field conductivity enhancement.

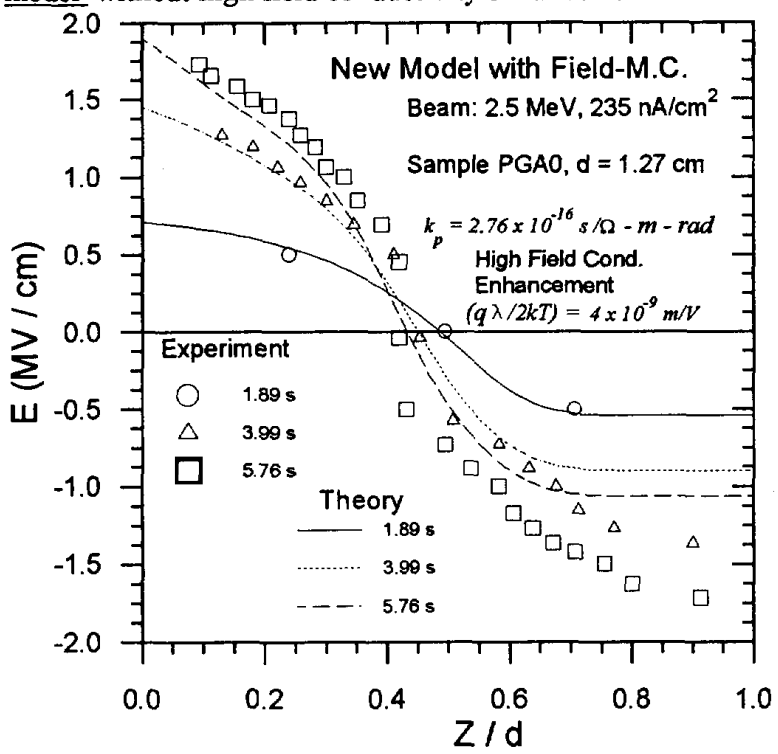

Fig. 7b. Electric field vs. distance for sample PGA0. New model. with high-field conductivity enhancement.

\section{COMPARISON OF THEORY AND EXPERIMENT FOR OTHER CASES}

Hikita et al. investigated a number of samples of PMMA of different thicknesses and with different electron beam energies and beam currents. We carefully modeled three other cases. In general, using the same conductivity parameters as in Section V, we found less, but reasonable, agreement between theory and experiment for the three other cases.

For example, for the case of a $1.6 \mathrm{MeV}$ beam incident on Sample PGA8, $d=0.635 \mathrm{~cm}$, calculations with the "old" model produced rather good agreement between the theoretical and experimental electric field in the front half of the sample. The old model predicts the electric field crossing point moving away from the incident surface with increasing irradiation time, in agreement with experiment in this one case. However, this experiment is exceptional, in that it is the only one out of some 20 experiments in which the crossing point moves away from the incident surface with increasing time, an anomalous result.

On the other hand, when calculations were made with the "new" model which included electric field effects on the fast electron motion, the field crossing point shifted toward the incident surface in the expected manner (see Fig. 8). The same RIC parameters used in Figs. $7 \mathrm{a}$ and $7 \mathrm{~b}$ produced reasonable agreement of magnitudes and shapes of the electric fields. Further comparison is unwarranted until new experiments can be performed as discussed next.

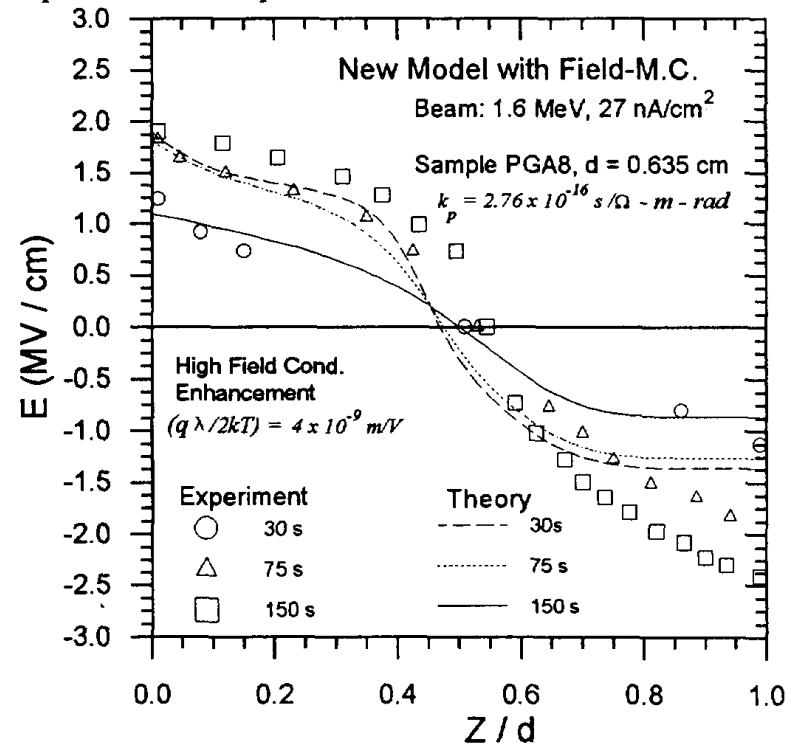

Fig. 8. Electric field vs. distance for sample PGA8. New model with high field conductivity enhancement.

\section{COMMENTS ON EXPERIMENTAL DATA}

The many experiments by Hikita indicate that:

a) the charge centroid nearly always moves toward the incident surface;

b) the charge profile forms a more narrow peak as time progresses; 
c) the system reaches steady state sooner than the old model predicts; and

d) the slope of the electric field at the entrance surface is less than the models without field enhancement would predict.

Our new model predicts all of these features.

It is necessary to match our theoretical model to the exact experimental conditions. With the extensive help of E. A. Beidl and M. Zahn of MIT, we investigated the earlier experiments[1]. As comparisons between our model and the experiments proceeded, we found a number of inconsistencies in the experimental data, as did E. A. Beidl[14].

The theoretical curves are consistent with the condition of grounded surfaces; i.e. the integral of the electric field over a path across the sample is equal to zero, yielding zero potential difference. However, the experimental electric fields do not indicate grounded surfaces. The integral of the experimental electric field values across the sample produces substantial voltage. This problem can be resolved by repeating these experiments with more attention devoted to the grounding of the surfaces.

We found that in about half the samples the total charge in the PMMA exceeded the time integral of the beam current density which was reported to have impacted the front of the samples. The apparatus for the measurement of electric field in PMMA is consistent from sample to sample. However, the incident beam current density was only indirectly measured, and the van de Graaff generator does not produce a highly stable beam. We therefore corrected the experimental measurement of the incident beam current density for each irradiation by matching the model to the temporal evolution of the electric fields at early times and small field.

The determination of RIC is difficult and not easily done based on the reported data set. For example, Fig. 12a of Ref. 1 shows that the total current decayed about $10 \%$ during the 100 second irradiation. The total current should be the incident current density times the area of the sample electrode. If the incident current is not constant, then the decay rate of the electrode current is not representative of the RIC alone. Additionally, the reported split faraday cup model is only approximate and not appropriate for accurately determining the power $\Delta$. Fig. $12 \mathrm{~b}$ of Ref. 1 uses data sets from different samples of various thicknesses and at different beam energies to determine the RIC power $\Delta$. There is an uncertainty of at least $20 \%$ in the measure of the beam current intensity, and thus the dose rate, in each measurement. Therefore we must allow for uncertainty in the experimentally determined RIC when comparing the experimental results with the model's predictions. A more appropriate method for measuring RIC parameters, which incorporates the complete modeling of charge currents in the insulator, has been reported[17] and could be used in future experiments of this nature.

We determined that the beam energies had a small but consistent and measurable error. At short times, before conduction current has redistributed much charge, the depth of zero electric field represents the mean depth of penetration of the incident beam electrons. Our early studies indicated that the measured depth of zero field was too shallow for 2.6 $\mathrm{MeV}$ electron beams. Subsequent recalibrations, using a multichannel analyzer and several nuclear sources, indicated that a $2.6 \mathrm{MeV}$ machine setting is close to $2.5 \mathrm{MeV}$ in reality. Therefore we vary the beam energy in the model to fully compare with the experimental results. The calibration at 1.6 $\mathrm{MeV}$ was found to be better than at $2.6 \mathrm{MeV}$.

\section{CONCLUSIONS AND SUGGESTIONS FOR FUTURE WORK}

\section{A. Conclusions}

We have developed a new method for calculating the time development of the electric field profile across a dielectric slab irradiated by 1.6 and $2.5 \mathrm{MeV}$ electrons. The combination of a Monte Carlo calculation including electric fields to calculate HE current and NUMIT to calculate total current yields improved predictions of electric field build-up in a dielectric slab as a function of time. This is important in cases where the incident beam energy is $\sim 100 \mathrm{keV}$ or above and the total dose exceeds $\sim 50 \mathrm{krads}$

The most pronounced effect of including the electric field in the Monte Carlo calculation is that the charge centroid (electric field "zero crossing-point") moves toward the incident surface instead of away from it, as is the case if the repulsive field effects on $\mathrm{HE}$ electron motion are neglected. This agrees with experimental data[1]

In addition, by comparing the model predictions with experimental data at several irradiation times, we have obtained values for the RIC and high-field enhanced conductivity. The combination of experimental data and the new model for the first time allows for the determination of high field conductivity enhancement in irradiated thick material without the requirement for application of very high voltage.

We believe that some of the differences between the model and experiment could be explained by inaccurate determination of the electron beam energy and current, incorrect values for the experimental total charge build-up in the dielectric slab, and failure of electrodes on the dielectric to be well grounded.

Another important finding of this research is that the dose, charge and current profiles differ significantly from those obtained by the simple application of codes such as ITS[10] to electron beam irradiation of insulating materials. If the sample is irradiated continuously, the charge build-up over time induces a significant internal electric field $(\sim 1-$ $2 \mathrm{MV} / \mathrm{cm}$ ) which has important implications for the dose, charge and current profiles. For incident energies above $\sim 100 \mathrm{keV}$, and for total dose exceeding $\sim 50 \mathrm{krads}$, application of a transport code, without taking into account the charge build-up in insulating materials will produce erroneous results. 


\section{B. Suggestions for Future Work}

Experiments similar to those of Hikita $e t$ al. should be repeated with well grounded surfaces. The original experiments were designed without the benefit of a model. We now have a theoretical model to aid in experiment design. We recommend further electric field measurements coupled with calculations using our model for immediate comparison and calibration checking.

There is a need for a faster algorithm to replace the long Monte Carlo calculations that must be performed at a number of time steps along the way. An improvement might be obtained by developing an empirical fit to the Monte Carlo data already obtained in order to give more rapid calculations of fast current and energy deposition profiles as a function of time for a specified beam current. The model would be applicable initially only to PMMA but should be able to make reasonable predictions for electron beam energies from 1-3 $\mathrm{MeV}$ and for a range of slab thicknesses.

\section{REFERENCES}

[1] M. Hikita, M. Zahn, K. A. Wright, C. M. Cooke and J. Brennan, "Kerr Electro-optic Field Mapping Measurements in Electron-beam Irradiated Polymethylmethacrylate," IEEE Trans. Electrical Insulation 23, 861-80, October 1988.

[2] A. R. Frederickson, "Charge Deposition, Photoconduction, and Replacement Current in Irradiated Multilayer Structures," IEEE Trans. Nuc. Sci., 22, 2556-61, Dec., 1975.

[3] S. Matsuoka, H, Sunaga, R. Tanaka, M. Hagiwara and K. Araki,"Accumulated Charge Profile in Polyethylene During Fast Electron Irradiations," IEEE Trans. Nuc. Sci., 23, 1447-52, 1976.

[4] O. B. Evdokimov and A. P. Yalovets, "General Problems of Fast-electron Transfer, Transfer in a Dielectric Plate for Nonuniform Electric Field," Izvestiya Vysshikh Uchebnykh Zavedenii, Fizika 10, 32-8, Oct. 1973.

[5] M. Kotera and H. Suga, "A Simulation of keV Electron Scatterings in a Charged-up Specimen," J. Appl. Phys. 63, 261-8, January, 1988.

[6] R. Tanaka, H. Sunaga and N. Tamura, "The Effect of Accumulated Charge on Depth Dose Profile in Poly(Methylmethacrylate) Irradiated with Fast Electron Beam," IEEE Trans. Nuc. Sci., 26, 4670-5, August 1979.

[7] A. R. Frederickson, "Radiation Induced Electrical Current and Voltage in Dielectric Structures," Air Force Cambridge Research Laboratories, Physical Sciences Research Papers, No. 613, AFCRL-TR-74-0582 (1974).

[8] T. Tabata and R. Ito, "An Algorithm for the Energy Deposition by Fast Electrons," Nucl. Sci. Eng., 53, 226239,1974
[9] M. J. Berger and R. Wang, "Multiple-Scattering Angular Deflections and Energy-Loss Straggling", Monte Carlo Transport of Electrons and Photons, Ch. 2, T. M. Jenkins, W. R. Nelson and A. Rindi, eds., Plenum Press, 1987.

[10] ITS 3.0 Code Package CCC-467, Radiation Shielding Information Center, Oak Ridge National Laboratory, Oak Ridge, TN, 1992.

[11]Y. V. Novozhilov and Y. A. Yappa, Electrodynamics, Ch. 6, Mir Publishers, Moscow, 1981.

[12] W. R. Nelson, H. Hirayama and D. W. O. Rogers, "The EGS4 Code System," Stanford Linear Accelerator Report SLAC-265 (1985).

[13] A. F. Bielajew, "Electron Transport in E and B Fields", Monte Carlo Transport of Electrons and Photons, Ch. 19, T. M. Jenkins, W. R. Nelson and A. Rindi, eds., Plenum Press, 1987.

[14]E. A. Beidl, "Modelling of Charge Deposition Profiles in Electron Beam Irradiated Dielectrics," M. Sc. Dissertation, Massachusetts Institute of Technology, Cambridge, MA, September 1992.

[15]B. Hilczer and J.. Malecki, Electrets, Elsevier, New York, NY, 1986.

[16] A. K. Jonscher, Dielectric Relaxation in Solids, Chelsea Dielectrics Press, 33 Lynwood Rd. London, 1983.

[17] A. R. Frederickson, "Radiation Induced Currents and Conductivity in Dielectrics," IEEE Trans. Nuc. Sci., 24, 2532-39, 1977.

\section{Acknowledgment}

The authors would like to acknowledge the extensive help in the analysis of the experimental data provided by Prof. M. Zahn and Mr. E. Beidl of the MIT Dept. of Electrical Engineering High Voltage Laboratory. 